\title{
The OPERA experiment: status and prospects
}

\author{
Marilisa De Serio* ${ }^{*}$ \\ Dip. Interateneo di Fisica Università di Bari, Via Amendola 173, 70126 Bari, Italy \\ E-mail: Marilisa.Deserio@ba.infn. it
}

Abstract: The next generation of long baseline experiments will be devoted to unambiguously confirm the existence of neutrino oscillations in the atmospheric sector. In this framework, a general description of the OPERA detector, its physics performance and status of construction is presented.

Solar neutrino experiments have recently obtained conclusive evidence[i]i] on neutrino flavour transitions observing solar $\nu_{e}$ conversion into active $\nu_{\mu}, \nu_{\tau}$ states. Atmospheric

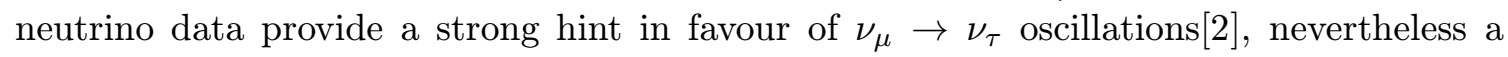
confirmation of the oscillation mechanism in the atmospheric sector, in particular the direct observation of $\nu_{\tau}$ appearance in a $\nu_{\mu}$ beam, is still missing. The next generation of long baseline accelerator experiments will provide a definite answer to the atmospheric $\nu$ anomaly problem, exploring the region of the oscillation parameter space $\left(\Delta m^{2}, \sin ^{2} 2 \theta\right)$ favored by Super-Kamiokande data.

OPERA [יivi] (Oscillation Project with Emulsion-tRacking Apparatus) will search for the appearance of $\nu_{\tau}$ 's in the CNGS $\nu_{\mu}$ beam from CERN SPS to Laboratori Nazionali del Gran Sasso (LNGS, Italy) over a baseline of $732 \mathrm{~km}$. Nuclear emulsions will be used as high resolution tracking detectors to directly observe the $\tau$ particles produced in $\nu_{\tau}$ charged current interactions within a massive lead target.

\section{The CNGS Beam}

The CERN Neutrino to Gran Sasso $\nu_{\mu}$ beam [4] has been designed to meet the requirements of a $\nu_{\tau}$ appearance experiment.

A $400 \mathrm{GeV}$ proton beam, accelerated by the CERN SPS, hits a 2 meters long segmented target, made of $10 \mathrm{~cm}$ long graphite rods. The $\pi$ and $K$ mesons produced as secondary particles are focused by a system of two coaxial lenses, the horn and the reflector, and conveyed towards a $1 \mathrm{~km}$ long decay tunnel. Two stations of silicon detectors, placed at the exit of the decay tunnel, monitor the beam transverse profile.

${ }^{*}$ Speaker.

${ }^{\dagger}$ On behalf of OPERA Collaboration. 
The main features of the CNGS beam are listed in table $i_{-i}^{-}$The average energy, about $17 \mathrm{GeV}$, is well above the $\tau$ lepton production threshold and the prompt contamination due to $\nu_{\tau}$ 's from $D_{s}$ decays is negligible.

\begin{tabular}{|l|c|}
\hline p.o.t./year & $4.5 \times 10^{19}$ \\
\hline$\nu_{\mu} /\left(m^{2}\right.$ p.o.t. $)$ & $7.78 \times 10^{-9}$ \\
\hline$\nu_{\mu} C C /($ p.o.t. $k t)$ & $5.85 \times 10^{-17}$ \\
\hline$<E>_{\nu_{\mu}}[\mathrm{GeV}]$ & 17 \\
\hline $\bar{\nu}_{\mu} / \nu_{\mu}$ & $2.1 \%$ \\
\hline$\nu_{e} / \nu_{\mu}$ & $0.8 \%$ \\
\hline $\bar{\nu}_{e} / \nu_{\mu}$ & $0.07 \%$ \\
\hline prompt $\nu_{\tau}$ & negligible \\
\hline
\end{tabular}

Table 1: The CNGS beam performance.

Possible upgrades of the PS/SPS machines are being extensively investigated in order to increase the intensity of the primary proton beam and/or improve the efficiency, thus increasing the neutrino flux. A possible scheme, that would yield a gain of a factor 1.46, has been already worked out currently going on in order to validate the feasibility of the project.

The civil engineering phase has been recently completed. The hadron stop was installed at the beginning of last September, the installation of the decay tube is ongoing and is expected to be completed by next summer. The CNGS project is on schedule, beam commissioning is foreseen in spring 2006 .

\section{The OPERA detector}

The layout of the OPERA detector is shown in fig.

Visual and electronic detection techniques have been combined to design a modular setup, made of two super-modules, each consisting of a target section plus a downstream muon spectrometer.

The basic target unit is a sandwich-like structure, called brick, made of 56 lead plates, $1 \mathrm{~mm}$ thick, and 57 interleaved nuclear emulsion foils. Each foil consists of two $42 \mu \mathrm{m}$ thick layers glued onto a $200 \mu \mathrm{m}$ plastic base, with transverse dimensions $\sim 12.7 \times 10.2 \mathrm{~cm}^{2}$. The brick thickness corresponds to $\sim 10 X_{0}$. Thanks to this type of structure, a segmented massive target $(\sim 1.8 \mathrm{kt})$ can be built, acting, at the same time, as a fine-grained detector that exploits the excellent tracking capabilities of nuclear emulsions (spatial resolution $<1 \mu \mathrm{m})$ to search for $\nu_{\tau}$ interaction vertices and subsequent $\tau$ decays.

Bricks are, in turn, assembled in planar walls, perpendicular to the beam direction. Each wall, namely a matrix of $52 \times 64$ bricks with transverse dimensions $\sim 6.7 \times 6.7 \mathrm{~m}^{2}$, is coupled to a pair of tracker planes, made of orthogonal plastic scintillator strips $(6.7 \mathrm{~m} \times 2.6 \mathrm{~cm} \times 1 \mathrm{~cm})$ readout by WLS fibres and 64-channel PMT's (8 per plane).

A wall and its electronic trackers constitute a module. The target section of each super-modules comprises 31 modules.

Each muon spectrometer consists of a dipolar magnet, made of two iron walls. A current of about $1200 \mathrm{~A}$ in the top and bottom coils produces a magnetic flux density of $1.55 \mathrm{~T}$ in the tracking region with vertical field lines of opposite directions in the two walls. In between the 12 slabs of each iron wall, bakelite RPC's are placed to track muons inside the magnet walls and measure the range of stopping muons. High resolution trackers, made of vertical drift tube planes, with an intrinsic resolution of $0.3 \mathrm{~mm}$ in the 


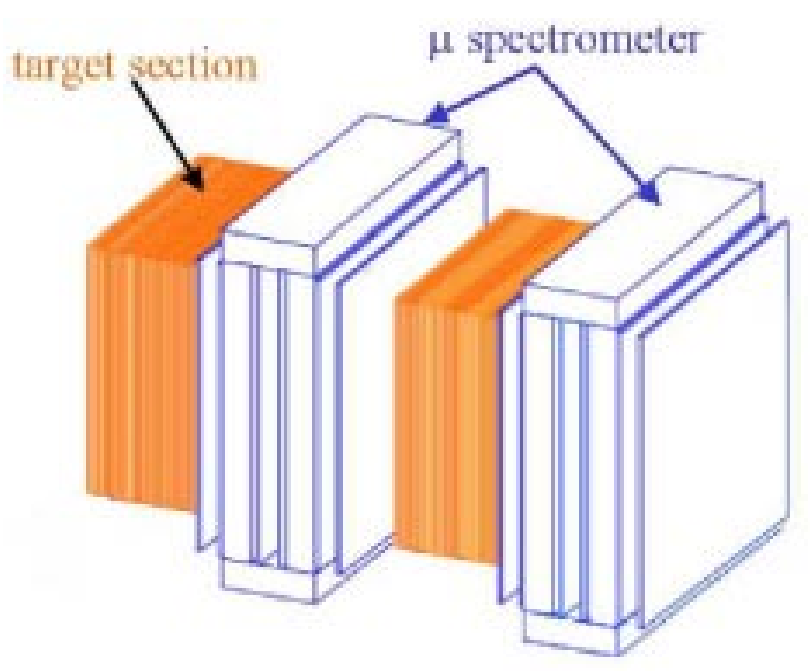

Figure 1: Layout of the OPERA detector.

bending direction, are placed in front, between and behind the magnet walls, resulting in a momentum resolution better then $20 \%$ over the entire kinematical domain of the experiment. The probability of wrong charge sign assignment, relevant to background rejection, is well below $0.5 \%$. The muon identification efficiency, obtained by combining spectrometer and scintillator tracker information, is greater than $95 \%$.

The installation of the first spectrometer in the Hall C at LNGS, where the experiment will run, is ongoing since May 2003. Recently, the first plane of RPC's has been installed.

The on-line analysis of electronic data (target tracker plus spectrometer) allows a realtime determination of the neutrino interaction position inside the target and a real-time identification of the brick where the interaction is likely to have occurred. The identified brick is then promptly removed from the target during the run. After processing, emulsions are sent to scanning stations/laboratories for vertex location, event selection, tau decay kink search, particle identification and kinematics.

\section{Nuclear emulsion scanning}

At least 30 neutrino interactions per day are expected to occur in the OPERA target. Correspondingly, the area of nuclear emulsions to be daily scanned is of the order of $10^{3} \mathrm{~cm}^{2}$.

The feasibility of an experiment such as OPERA mainly relies on the significant improvements recently achieved in the development of automatic scanning systems. Computer controlled microscopes are able to perform 3D reconstruction of particle tracks recorded in emulsion, starting from a sequence of $2 \mathrm{D}$ tomographic images of each field of view, taken at different focal depths by means of high resolution cameras.

Two R\&D projects are currently in progress within the OPERA Collaboration, aiming at the development of very high speed systems, able to scan an area $>20 \mathrm{~cm}^{2}$ in 1 hour. 

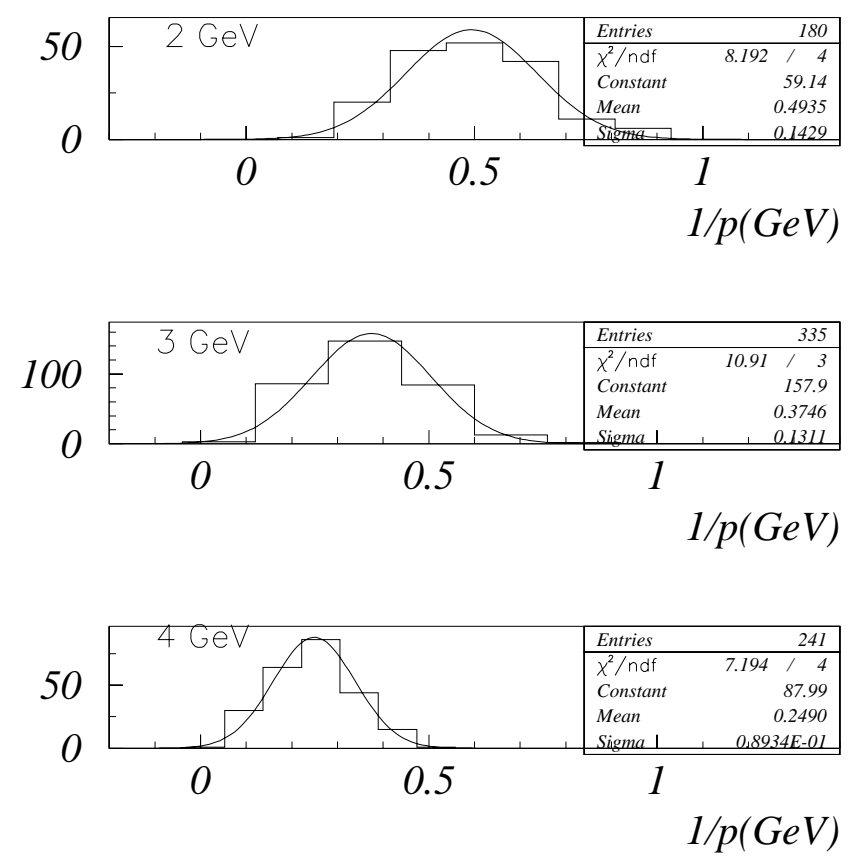

Figure 2: Momentum reconstruction of 2,3 and $4 \mathrm{GeV} / \mathrm{c}$ pions in a $3 X_{0}$ brick.

The Japanese project $S$-UTS is an evolution of the real-time processor Track Selector [6i]", invented by the University of Nagoya. Key features of the system are a very high frame rate CCD camera, working at $3 \mathrm{kHz}$, and a piezo-controlled objective lens. The movement of the z-axis is synchronous with that of the xy stage, so that image-taking can be performed whilst the stage is in motion.

A European project, involving groups from several institutions, aims to develop a software-based system with similar performances, that makes use of customized commercial

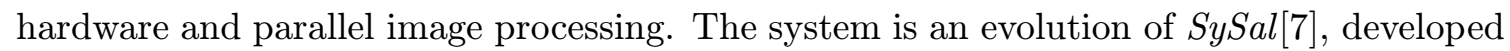
by the University of Salerno (Italy).

The track of a minimum ionising particle is visible in a developed emulsion as a sequence of black grains, with a diameter of $\sim 0.8 \mu \mathrm{m}$, aligned along the particle direction. The sensitivity of a typical emulsion film is of about $30 \div 40$ grains per $100 \mu \mathrm{m}$. The intrinsic position resolution, measured as the average residual of grain centres with respect to fitted trajectory, is $\sim 0.06 \mu \mathrm{m}$. In fact, the achievable resolution is limited by current readout accuracy to $0.3 \mu \mathrm{m}$. The resulting angular resolution is $\simeq 2 \mathrm{mrad}$, adequate to detect $\tau$ decay topology.

Thanks to the sub-micron accuracy, momentum measurements by multiple Coulomb scattering can be accomplished. The results of a test experiment held at CERN PS (T9 beam-line) are shown in fig. ㄱ. A $3 X_{0}$ brick was exposed to 2,3 and $4 \mathrm{GeV} / \mathrm{c}$ pions. Momentum resolutions ranging from $28 \%$ to $36 \%$ were obtained [i $[\bar{i}]$.

The multiple scattering technique can also be applied to identify electrons. Electrons loose a significant fraction of their energy by bremsstrahlung while traversing a brick. Differently, the energy of hadrons remains practically constant. A $\chi^{2}$ analysis can be applied to compare measured deflections with expected angular deviations according to 


\begin{tabular}{|c|c|c|}
\hline$\Delta m^{2}=1.3 \times 10^{-3} \mathrm{eV}^{2}$ & $\Delta m^{2}=2.0 \times 10^{-3} \mathrm{eV}^{2}$ & $\Delta m^{2}=3.0 \times 10^{-3} \mathrm{eV}^{2}$ \\
\hline $3.1(4.7)$ & $7.3(11.0)$ & $16.4(24.6)$ \\
\hline
\end{tabular}

Table 2: Expected signal events in 5 years of data-taking assuming nominal CNGS configuration and a higher intensity $(\times 1.46$, in brackets $)$.

multiple scattering theory for each given value of the incident energy, treated as a free parameter of the fit. This method, complemented by shower signal detection, was applied to a test experiment performed at CERN PS (T7 beam-line) in 2001. A $5 X_{0}$ brick was exposed to 2 and $4 \mathrm{GeV} / \mathrm{c}$ electron-pion beams. An identification efficiency of $\sim 90 \%$ was found [iפ⿴囗十)

\section{Oscillation search}

The search for $\nu_{\mu} \rightarrow \nu_{\tau}$ oscillations is performed by looking for the $\tau^{-}$particle in the final state of the $\mathrm{CC}$ process $\nu_{\tau} N \rightarrow \tau^{-} X$ through its characteristic decay topology $(k i n k)$ into muon $(B . R . \simeq 17.4 \%)$, electron $(B . R . \simeq 17.8 \%)$ and single charged hadron $(B . R . \simeq 49.5 \%)$.

Two different strategies are applied to select candidate events, depending on the decay type. Short decays, namely decays occurring in the same lead plate as the primary $\nu$ interaction, are identified through the detection of a large impact parameter track with respect to a reconstructed vertex. Long decays, occurring in the downstream emulsion foil or lead plate with respect to the $\nu$ interaction point, are selected by looking for an angular mismatch.

Table 2 shows the numbers of expected events in 5 years of run $\left(2.25 \times 10^{20}\right.$ p.o.t. $)$ as a function of $\Delta m^{2}$ assuming maximal mixing. The $\Delta m^{2}$ values correspond to the latest SK $90 \%$ C.L. interval [i $\left.{ }_{1}^{1} \overline{0}\right]$. The values reported in brackets refer to an integrated primary proton intensity of $3.28 \times 10^{20}$ p.o.t. (nominal intensity $\times 1.46$ ). At $\Delta m^{2}=2.0 \times 10^{-3} \mathrm{eV}^{2}$ (SK best fit value), the expected signal is 7.3 (11.0) events, corresponding to an overall $\tau$ detection efficiency of $9.1 \%$. The total number of background events, due to charm production and decay with unidentified primary muon, hadron re-interactions and largeangle muon scattering in lead, is 0.7 (1.1).

In case no $\tau$ candidate is found, OPERA would exclude the oscillation hypothesis at $90 \%$ C.L. for $\Delta m^{2}<1.2 \times 10^{-3} \mathrm{eV}^{2}$.

The experiment potential to search for $\nu_{\mu} \rightarrow \nu_{e}$ oscillations and the sensitivity to $\theta_{13}$ have also been investigated [i] i i i $]$.

Studies to improve the $\tau$ detection efficiency and reduce the background are ongoing.

\section{Conclusions}

The construction of the OPERA detector and its installation in the Hall C at LNGS are in progress. The experiment is expected to start running in spring 2006, when the 
commissioning of the CNGS beam is foreseen.

At the present status of the analysis, OPERA is expected to observe 7.3 signal events at the current SK $\Delta m^{2}$ best fit value and, correspondingly, 0.7 background events.

Studies to increase the CNGS neutrino flux are ongoing. A possible solution envisages an increase of the primary proton intensity of a factor 1.46. This scheme would result in 11.0 detectable $\nu_{\tau}$ 's and 1.1 background events.

\section{References}

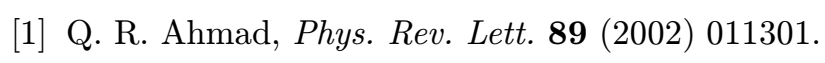

[2] Y. Fukuda et al., Phys. Rev. Lett. 81 1998$) 1562$

M. H. Ahn et al., IPhys. Rev. Lett. 90 $\mathbf{9} 2003) 041801$

[3] M. Guler et al., CERN-SPSC 2000-028;

M. Guler et al., CERN-SPSC 2001-025.

[4] A. E. Ball et al., CERN-SL/2000-063 EA;

M. Clément et al., CERN-SL/2002-012.

[5] R. Cappi et al., CERN-SL/2001-032.

[6] A. Aoki et al., 'Nucl. Instrum. Meth. B51 1990$) 466$.

[7] G. Rosa et al., 'Nucl. Instrum. Meth. Á394 1997$)$ 35t.

[8] M. De Serio et al., №cl. Instrum. Meth. A $\mathbf{5 1 2}(2003) 539_{*}^{\prime}$

[9] K. Kodama et al., 'Rev. Sci. Instrum.

[10] Y. Hayato, talk at EPS2003, http://eps2003.physik.rwth-aachen.de.

[11] M. Komatsu et al., $\bar{J}$. Phys. $\mathbf{G} 29$ 